\title{
Polariton Evaporation: The Blackbody Radiation Nature of the Low-Frequency Radiation Emitted by Radiative Polaritons to the Surrounding Space
}

\author{
Yosyp Schwab, Harkirat S. Mann, Brian N. Lang, Giovanna Scarel* \\ Department of Physics and Astronomy, James Madison University, Harrisonburg, USA \\ Email: scarelgx@imu.edu
}

Received 19 February 2014; revised 23 March 2014; accepted 8 April 2014

Copyright $(2014$ by authors and Scientific Research Publishing Inc.

This work is licensed under the Creative Commons Attribution International License (CC BY).

http://creativecommons.org/licenses/by/4.0/

(c) (i) Open Access

\begin{abstract}
Upon formation, radiative polaritons in thin oxide films or crystals emit radiation to the surrounding space. This radiation is confined in a small range of the microwave to far-infrared region of the electromagnetic spectrum, independently of the oxide chemistry. This work shows that the low-frequency radiation is blackbody radiation associated with a temperature directly related to the boson character of the radiative polaritons and to their amount. The proximity of this temperature to absolute zero Kelvin explains the confinement of the frequency. This phenomenon is named polariton evaporation.
\end{abstract}

\section{Keywords}

Polaritons, Dielectrics, Thin Films, Infrared Spectroscopy

\section{Introduction}

Radiative polaritons (RPs) were discovered in the late sixties [1]-[3] and recently gained attention due to their ability to explain optical and thermal properties of thin oxide films or crystals [4]-[11]. Radiative polaritons form upon the absorption of photons from infrared (IR) radiation by phonons in thin oxide films or crystals. The coupling occurs when photons and phonons oscillate at the same frequency. Unlike surface phonon-polaritons [12], whose frequency is a real number, RPs are characterized by a complex angular frequency $\omega=\omega_{r}+I \omega_{i}$, where $I$ is the imaginary unit and the subscripts $r$ and $i$ refer to the real and imaginary parts, respectively

${ }^{*}$ Corresponding author.

How to cite this paper: Schwab, Y., et al. (2014) Polariton Evaporation: The Blackbody Radiation Nature of the Low-Frequency Radiation Emitted by Radiative Polaritons to the Surrounding Space. World Journal of Condensed Matter Physics, 4, 58-65. http://dx.doi.org/10.4236/wjcmp.2014.42009 
[3]. The real part, $\omega_{r}$, is the resonant frequency, and is larger than the imaginary part, $\omega_{i}$ [13]. Additionally, through IR absorption spectra, $\omega_{r}$ provides the central frequency of the absorption peak, while $\omega_{i}$ supplies the spread of the absorption peak around $\omega_{r}$ [2] [3] [13], or half of the peak width. It was recently found that the frequency $\omega_{i}$ corresponds to the frequency of the experimentally observed low-frequency radiation emitted to the space surrounding the RP's formation site [13]. Such radiation lasts as long as the exciting IR radiation illuminates the targeted thin oxide film or crystal. The existence of such low-frequency radiation, however, is so far only viewed as the consequence of the presence of $\omega_{i}$ in one of the exponential terms in the expression for the polarization $\boldsymbol{P}(x, t)$ :

$$
\boldsymbol{P}(x, t) \propto \mathrm{e}^{I\left(k_{x r} x-\omega_{r} t+\mathrm{i} k_{x i} x-i \omega_{i} t\right)}=\mathrm{e}^{I\left(k_{x r} x-\omega_{r} t\right)} \mathrm{e}^{-\left(k_{x i} x-\omega_{i} t\right)},
$$

where $k_{x}$ is a component of the complex wave-vector $\boldsymbol{k}$ parallel to the thin oxide film or crystal surface [2] [12]. The term $\mathrm{e}^{-\left(k_{x i} x-\omega_{i} t\right)}$ in Equation (1) generates a wave propagating away from the site of the thin oxide film or crystal that generated the RP without decay. This description gives merely a mathematical explanation without providing a physical mechanism of the origin of the low-frequency radiation with frequency $\omega_{i}$. Furthermore, no explanation is provided for the wide span of the $\omega_{r}$ values, on one hand, and the confinement of the $\omega_{i}$ values in a small frequency range in the microwave to far-IR region for a large variety of thin oxide films in a broad thickness range. The goal of this work is to unveil the explanation (1) of the physical mechanism underpinning the formation of the low-frequency radiation with frequency $\omega_{i}$, and (2) for the wide span of the $\omega_{r}$ values, on one hand, and the confinement of the $\omega_{i}$ values. The importance of this effort is in its ability to elucidate whether a new source of radiation accompanies the formation of RPs, or if a connection can be found with already known phenomena.

\section{Experimental Data and Simulation Method}

The experimental data consist of the IR spectroscopic information on thin oxide films reported by previous research. From IR absorption spectra, the frequency $\omega_{r}$ can be derived from the centroid of the absorption peak, while the frequency $\omega_{i}$ is derived from half of the width of the absorption peak [2] [3] [13], as illustrated in Figure 1. Table 1 summarizes the findings obtained for the 0TH type RP [2] [3] of various oxides, such as $\mathrm{TiO}_{2}$, $\mathrm{La}_{2} \mathrm{O}_{3}, \mathrm{Al}_{2} \mathrm{O}_{3}$, and $\mathrm{Lu}_{2} \mathrm{O}_{3}[13]$-[17]. It is found that, for the 0TH type RP, the $\omega_{r}$ values span in $a \approx 450 \mathrm{~cm}^{-1}$ wide range, while the corresponding $\omega_{i}$ values span in a range $\approx 35 \mathrm{~cm}^{-1}$ wide. The range of the $\omega_{i}$ values is $\approx 8 \%$ that of the $\omega_{r}$ values. In addition, the $\omega_{r}$ values are very characteristic of a peculiar crystal structure

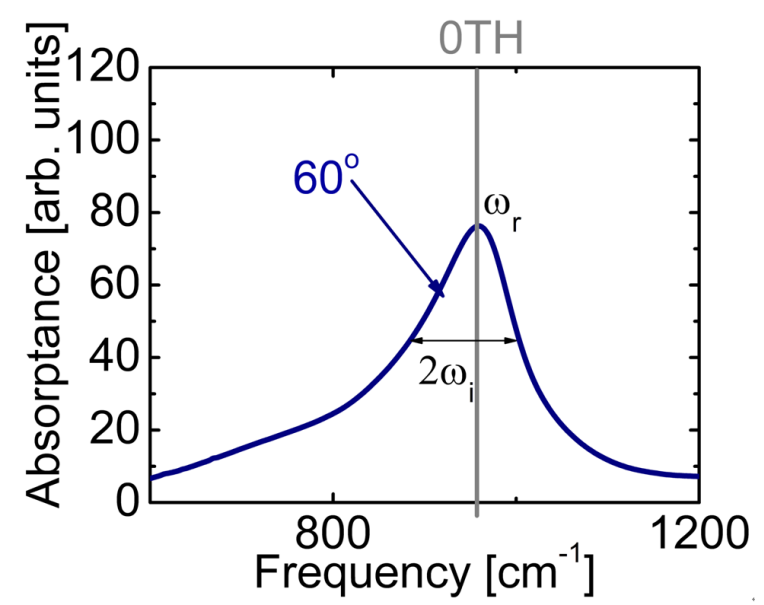

Figure 1. Experimental absorptance spectra at a $60^{\circ}$ incidence angle for a $250 \mathrm{~nm}$ thick $\mathrm{Al}_{2} \mathrm{O}_{3}$ film on $\mathrm{Si}(100)$ measured in reflection mode [13]. The 0TH type RP is labeled with the frequency $\omega_{r}$ at the centroid of the absorption peak, and the frequency $2 \omega_{i}$ indicating the width of the absorption peak [13]. 
Table 1. Values of the real part, $\omega_{r}$, and of the imaginary part, $\omega_{i}$, of the complex angular frequency $\omega=\omega_{r}+I \omega_{i}$ of the OTH type RP, where $I$ is the imaginary unit, for various oxide thin films at various thicknesses. The chemical potential $\mu$ is estimated in $J$ for $\left\langle n_{R P}\right\rangle=1$ mole, and room temperature $\left(293 \mathrm{~K}\right.$, or $\left.20^{\circ} \mathrm{C}\right)$.

\begin{tabular}{cccccc}
\hline \multicolumn{2}{c}{ Oxide film } & Thickness $[\mathrm{nm}]$ & $\omega_{r}\left[\mathrm{~cm}^{-1}\right]$ & $\omega_{i}\left[\mathrm{~cm}^{-1}\right]$ & Chemical potential $[\mathrm{J}]$ \\
\hline $\mathrm{La}_{2} \mathrm{O}_{3}$ & {$[15]$} & 250 & 546 & 50 & $80.62 \times 10^{-22}$ \\
$\mathrm{TiO}_{2}$ & {$[14]$} & 250 & 850 & 40 & $140.25 \times 10^{-22}$ \\
$\mathrm{Al}_{2} \mathrm{O}_{3}$ & {$[16]$} & 250 & 968 & 39 & $164.97 \times 10^{-22}$ \\
$\mathrm{Lu}_{2} \mathrm{O}_{3}$ & {$[17]$} & 40 & 611 & 16 & $93.14 \times 10^{-22}$ \\
$\mathrm{TiO}_{2}$ & {$[13]$} & 50 & 836 & 19 & $137.60 \times 10^{-22}$ \\
\hline
\end{tabular}

[1] [3] [18], which can be determined using IR spectroscopy. The $\omega_{i}$ values, instead, are confined in the microwave to far-IR region of the electromagnetic frequency spectrum [13], as shown in Table 1, for a large variety of oxides and in a broad thickness range.

In this research, the spectroscopic data collected in Table 1 are used and elaborated according to the hypothesis formulated in the following section (Section 3 ).

\section{Hypothesis}

For a large variety of thin oxide films in a broad thickness range, the understanding of the wide span of the $\omega_{r}$ values, on one hand, and of the confinement, on the other, of the $\omega_{i}$ values requires a new approach beyond the analysis of the polarization $\boldsymbol{P}(x, t)$, as done in previous research [2] [13]. The alternative path chosen here is of statistical mechanics nature. It involves photons and phonons, which are mass-less bosons obeying the general Bose-Einstein statistics:

$$
\left\langle n_{j}\right\rangle=\frac{1}{\mathrm{e}^{\frac{1}{k_{B} T}\left(\varepsilon_{j}\right)}-1} .
$$

Here, $\left\langle n_{j}\right\rangle$ is the occupation number of bosons with energy $\varepsilon_{j}, k_{B}=1.381 \times 10^{-23} \frac{\mathrm{J}}{\mathrm{K}}$ the Boltzmann constant, and $T$ the temperature. Stemming from the coupling between photons and the phonons, RPs must be bosons. The formation of a RP can be described as the result of an annihilation operator $a$ applied to both the Hamiltonians of the photon and phonon, and contemporarily a creation operator $a^{+}$applied to the Hamiltonian of the RP. Alternatively, the effort made by a thin oxide film or crystal to couple photons and phonons and generate a RP can be expressed in terms of the chemical potential $\mu$. In this context, $\mu$ is thus defined as the free energy needed to rise or lower the number or moles of RPs in a thin oxide film or crystal. Assuming that $\varepsilon_{j}=\hbar \omega$ is the energy of a RP, and considering $\left\langle n_{R P}\right\rangle$ as the number of moles of RPs, the Bose-Einstein statistics for RPs is:

$$
\left\langle n_{R P}\right\rangle=\frac{1}{\mathrm{e}^{\frac{1}{k_{B} T}(\hbar \omega-\mu)}-1},
$$

where $\hbar$ is the reduced Planck's constant $\left(1.05457 \times 10^{-34} \mathrm{Js}\right)$. It follows that the temperature $T$ associated to the RP can be derived from Equation (3) as:

$$
T=\frac{1}{k_{B}} \frac{(\hbar \omega-\mu)}{\ln \left(1+\frac{1}{\left\langle n_{R P}\right\rangle}\right)},
$$

where $1+\frac{1}{\left\langle n_{R P}\right\rangle} \geq 1, \hbar \omega-\mu>0$, and $\mu$ is real and positive. Furthermore, since $\omega$ is complex, the temper- 
ature $T$ must be complex too. The real part:

$$
T_{r}=\frac{1}{k_{B}} \frac{\left(\hbar \omega_{r}-\mu\right)}{\ln \left(1+\frac{1}{\left\langle n_{R P}\right\rangle}\right)},
$$

is related to the frequency of the radiation absorbed at $\omega_{r}$ and to the chemical potential $\mu$, whereas the imaginary part:

$$
T_{i}=\frac{1}{k_{B}} \frac{\left(\hbar \omega_{i}\right)}{\ln \left(1+\frac{1}{\left\langle n_{R P}\right\rangle}\right)},
$$

is associated with the low-frequency radiation emitted at frequency $\omega_{i}$. For both the real and the imaginary parts, Equation (3) links $T$ to $\left\langle n_{R P}\right\rangle$, the number of moles of RPs, and to the magnitude of the characteristic RPs frequencies, $\left|\omega_{r}\right|$ and $\left|\omega_{i}\right|$ respectively. The real and an imaginary temperatures $T_{r}$ and $T_{i}$ coexist in a system of thin oxide films or crystals, and their environment, while accompanying RP's formation. The investigation will now proceed with discussing the physical meaning of $T_{r}$ and $T_{i}$, and their relationship with the origin and properties of the low-frequency radiation emitted at frequency $\omega_{i}$.

\section{Results and Discussion}

Equation (5) provides an expression for the real temperature, $T_{r}$, whose nature needs to be disclosed alongside the value of the chemical potential $\mu$. Two possibilities can be considered. One is that the value of the chemical potential $\mu$ is the same for the RPs in all types of a thin oxide film or crystal that can support them. In this case, according to Equation (5), $T_{r}$ depends on the oxide chemistry, thus on $\omega_{r}$. This picture rules out the influence on the results due to the laboratory temperature, which determines the number of moles for RPs $\left\langle n_{R P}\right\rangle$ at a given frequency [19], and will therefore be neglected. The alternative possibility is that the value of the chemical potential $\mu$ varies for different types of thin oxide films or crystals. In this case, $T_{r}$ can be constant, and taken as the laboratory temperature at which the RPs were generated. The data in Table 1 were collected at $293 \mathrm{~K}$, or $20^{\circ} \mathrm{C}$, which is thus assumed as the laboratory's temperature. In this case, the chemical potential $\mu$ values vary depending upon the thin oxide films considered, and their values in $J$ are reported in Table 1 assuming $\left\langle n_{R P}\right\rangle=1$. Low $\mu$ values characterize a crystal structure and chemistry which require a small amount of energy to promote RPs formation. The span in the $\mu$ values explains the large span of the $\omega_{r}$ values measured for thin oxide films. The trend of the chemical potential $\mu$ versus $\omega_{r}$ :

$$
\mu=\hbar \omega_{r}-k_{B} T_{r} \ln \left(1+\frac{1}{\left\langle n_{R P}\right\rangle}\right),
$$

is illustrated in Figure 2. It can be observed that larger chemical potential $\mu$ values are associated to larger absorption frequencies $\omega_{r}$. The trends are similar for $\left\langle n_{R P}\right\rangle=1$ and $\left\langle n_{R P}\right\rangle=0.5$ with $\omega_{r}$ values ranging between $500 \mathrm{~cm}^{-1}$ and $1000 \mathrm{~cm}^{-1}$, as observed in experimental spectra.

Equation (6) gives the expression for the imaginary temperature $T_{i}$. This expression is similar to

$$
T_{\text {blackbody }}=\frac{\hbar \omega_{i}}{2.82 k_{B}}[20] \text {, the temperature of blackbody radiation with maximum spectral radiance } I_{\omega} \text { at } \omega_{i} \text {. }
$$

The factor 2.82 stems from the maximization of $I_{\omega}=\frac{2 \pi \hbar \omega^{3}}{c^{2}} \frac{1}{\mathrm{e}^{\frac{\hbar \omega}{k_{B} T}}-1}$ with respect to $\omega$ [20], where $c$ is the speed of light in vacuum. The similarity between Equation (6) and $T_{\text {blackbody }}$ suggests two possible consequences. One is that that the radiation emitted to the space surrounding the RP's formation site at frequency $\omega_{i}$ is blackbody radiation. Thus, the association between $\omega_{i}$ and the imaginary temperature $T_{i}$ in Equation (6) is named polariton evaporation, which can be viewed as the emission of energy, or as some kind of dissipation phenomenon, related to the frequency $\omega_{i}$. Stated differently, the dissipated RP's energy $\hbar \omega_{i}$ plus the energy from the IR radiation absorbed around frequency $\omega_{r}$ and transformed into heat [21], give the energy of the IR 


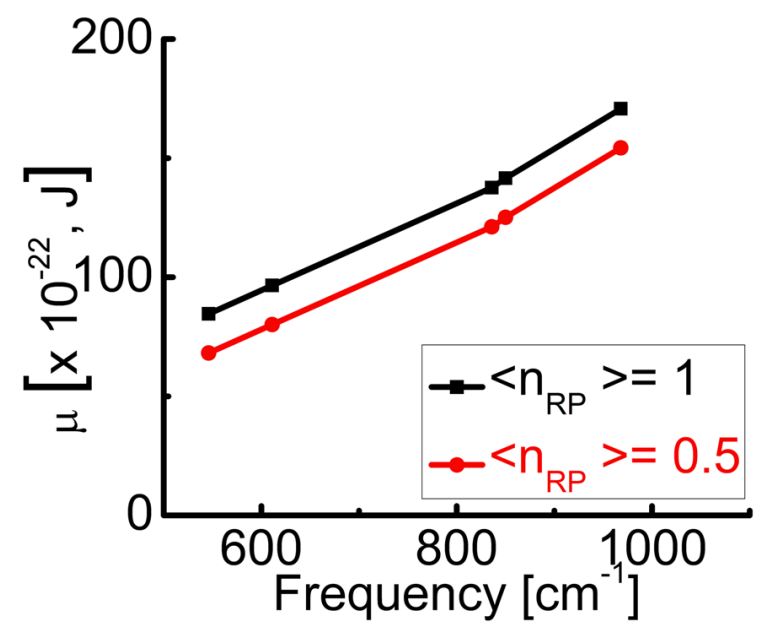

Figure 2. The chemical potential $\mu$ versus frequency $\omega_{r}$ evaluated numerically for $\left\langle n_{R P}\right\rangle=1$ mole (filled squares), and for $\left\langle n_{R P}\right\rangle=0.5$ mole (filled circles). Room temperature $\left(293 \mathrm{~K}\right.$, or $\left.20^{\circ} \mathrm{C}\right)$ is assumed in the evaluation. The symbols correspond to the experimentally determined frequencies $\omega_{r}$ of the 0TH type RP for thin oxide films of $\mathrm{TiO}_{2}, \mathrm{La}_{2} \mathrm{O}_{3}, \mathrm{Al}_{2} \mathrm{O}_{3}$, and $\mathrm{Lu}_{2} \mathrm{O}_{3}$ at thicknesses specified in Table 1.

photons absorbed by the thin oxide film or crystal. The other consequence is that, for $T_{i}=T_{\text {blackbody }}$,

$$
2.82=\ln \left(1+\frac{1}{\left\langle n_{R P}\right\rangle}\right) .
$$

Equation (8) has a solution for $\left\langle n_{R P}\right\rangle=0.06$. Since here the magnitude of $\left\langle n_{R P}\right\rangle$ is a fractional value, this quantity should not be related to number of particles. Rather, defining $\left\langle n_{R P}\right\rangle$ as the mole number of RPs seems more appropriate. With this assumption, the trend of $T_{i}$ versus $\omega_{i}$ between $25 \mathrm{~cm}^{-1}$ and $50 \mathrm{~cm}^{-1}$ is illustrated in Figure 3 for $\left\langle n_{R P}\right\rangle=1$ and $\left\langle n_{R P}\right\rangle=0.5$. The $T_{i}$ temperature values increase linearly and with positive slope with respect to $\omega_{i}$. The values of $T_{i}$ are around or below $73 \mathrm{~K}$, or $-200^{\circ} \mathrm{C}$, and vary by only $40^{\circ} \mathrm{C}$ to $20^{\circ} \mathrm{C}$ for $\omega_{i}$ between $25 \mathrm{~cm}^{-1}$ and $50 \mathrm{~cm}^{-1}$ for $\left\langle n_{R P}\right\rangle=1$ and $\left\langle n_{R P}\right\rangle=0.5$, respectively. Further more, for $\left\langle n_{R P}\right\rangle=1$ the slope is $2.0 \frac{{ }^{\circ} \mathrm{C}}{\mathrm{cm}^{-1}}$, while for $\left\langle n_{R P}\right\rangle=0.5$ it is $1.2 \frac{{ }^{\circ} \mathrm{C}}{\mathrm{cm}^{-1}}$. The dependence of the $T_{i}$ values to the number of moles is related to the dependence of the $\omega_{i}$ values to film thickness, or the amount of material in the thin oxide film, as discussed in previous research [13]. The proximity of the $T_{i}$ values to $0 \mathrm{~K}$, or $-273{ }^{\circ} \mathrm{C}$, combined with the effects of the number of moles of the thin oxide film, confines the $\omega_{i}$ values in a small range in the microwave to far-IR region of the electromagnetic frequency spectrum [13], as shown in Table 1. This observation also signifies that detecting $T_{i}$ is not trivial: it would require a thermometer operating near $0 \mathrm{~K}$, and sensitive to very small variations of temperature.

\section{Comparison with Other "Evaporation" Phenomena}

Evaporation phenomena and imaginary temperature in the available literature are now discussed. Evaporation phenomena in particular are not isolated, and one of the most popular is black hole evaporation [22] [23]. The emission of the so-called Hawking radiation was hypothesized after the discovery that black holes have entropy, and thus temperature. Because of the temperature, black holes must radiate blackbody radiation. Unlike the polariton evaporation case, the temperature of black holes was discovered before the radiation. The temperature of the black hole is a real, not an imaginary quantity [22] [23]. The evaporation phenomenon for black holes is of quantum-mechanical nature, and involves the "evaporation" of mass [23] in the form of particle production via 


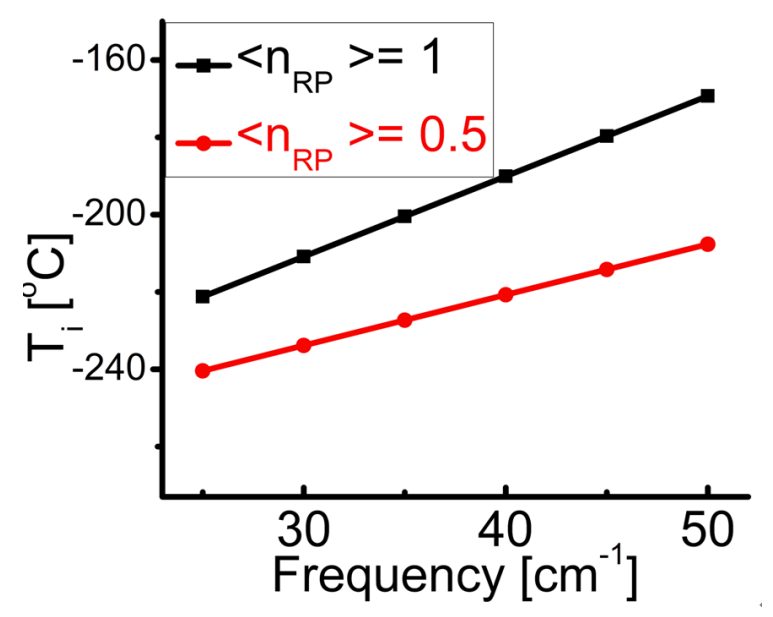

Figure 3. The imaginary temperature $T_{i}$ versus fre-
quency $\omega_{i}$ evaluated numerically for $\left\langle n_{R P}\right\rangle=1$ mole
(filled squares), and for $\left\langle n_{R P}\right\rangle=0.5$ mole (filled circles).
The frequency values cover the range in which $\omega_{i}$ is
found in experimental IR spectra of $\mathrm{TiO}_{2}, \mathrm{La}_{2} \mathrm{O}_{3}, \mathrm{Al}_{2} \mathrm{O}_{3}$,
and $\mathrm{Lu}_{2} \mathrm{O}_{3}$ oxide films as specified in Table 1 .

tunneling [24]. Polariton evaporation, on the other hand, is the consequence of a complex frequency, which results in a complex temperature. Thus, even if polariton evaporation is not accompanied by real mass loss, the complex frequencies and temperature are related to the existence of dissipation events [13] [25]. The energy balance is such that the sum of the energy $\hbar \omega_{i}$ dissipated by the RPs plus the energy from the IR radiation absorbed around frequency $\omega_{r}$ and transformed into heat [21], are equal the energy of the IR photons irradiating the thin oxide film or crystal. Similarly to the case of the imaginary temperature $T_{i}$ of polariton evaporation, which is relatively low $(\sim 50 \mathrm{~K})$, the temperature of the black hole is predicted to be low $\left(\approx 2 \times 10^{-14} \mathrm{~K}\right.$ for a supermassive black hole) [26]. Investigations are under way to detect the existence of the Hawking radiation, whose signature is contained in gamma-ray bursts [23]. On the other hand, the radiation related to polariton evaporation was recently detected [13]. Finally, similarly to the temperature of the black holes which depends on $\frac{1}{M_{b h}}$, where $M_{b h}$ is the mass of the black hole [23], the $T_{i}$ of RPs depends onthe amount of RPs in the form of their number of moles $\left\langle n_{R P}\right\rangle$, as shown in Figure 3. Even with all the differences reported, polariton and black hole evaporation are both related to blackbody radiation, showing a deep unity in microscopic and macroscopic physical and natural phenomena.

The concept of imaginary temperature is not as popular as that of "evaporation". The literature is short of examples of imaginary temperatures deriving from mathematically complex quantities. On the other hand, the expression of imaginary temperature is used most notably in the biological field, where it is defined as the "statistical value for a thermostat made of particles with real mass" [27]. Mathematically imaginary quantities, however, are normally related to dissipation [13] [25].

\section{Summary and Significance}

Because of their bosonic nature, radiative polaritons have a temperature associated with the low frequency radiation they emit to the space surrounding their formation site. The radiation is due to blackbody radiation associated with a temperature which stems from the imaginary part of the complex frequency of radiative polaritons and is related to their amount. The relationship with blackbody radiation aids in explaining the confinement of the $\omega_{i}$ values in a narrow frequency interval in the microwave to far-infrared region. The $\omega_{r}$ values span in a wide frequency range because they are related to the oxide film and crystal properties, as illustrated here specifically for the $0 \mathrm{TH}$ type radiative polariton in Table 1. Finally, polariton evaporation resembles black hole evaporation, giving insight on the profound unity among physical phenomena in the nano- and the macro-scale. 


\section{Acknowledgements}

This work was supported by the Thomas F. Jeffress and Kate Miller Jeffress Memorial Trust (grant \# J-1053), the James Madison University (JMU) Center for Materials Science, the JMU Department of Physics and Astronomy, and the NSF-REU and Department of Defense ASSURE program (grant \#0851367). The authors thank Profs. K. Fukumura, A. Constantin, C. S. Whisnant, and J. C. Zimmerman (JMU) for fruitful discussions.

\section{References}

[1] Berreman, D.W. (1963) Infrared Absorption at Longitudinal Optic Frequency in Cubic Crystal Films. Physical Review, 130, 2193-2198. http://dx.doi.org/10.1103/PhysRev.130.2193

[2] Kliewer, K.L. and Fuchs, R. (1966) Optical Modes of Vibration in an Ionic Crystal Slab including Retardation. II. Radiative Region. Physical Review, 150, 573-588. http://dx.doi.org/10.1103/PhysRev.150.573

[3] Fuchs, R., Kliewer, K.L. and Pardee, W.J. (1966) Optical Properties of an Ionic Crystal Slab. Physical Review, 150, 589-596. http://dx.doi.org/10.1103/PhysRev.150.589

[4] Scarel, G., Na, J.-S., Gong, B. and Parsons, G.N. (2010) Phonon Response in the Infrared Region to Thickness of Oxide Films Formed by Atomic Layer Deposition. Applied Spectroscopy, 64, 120-126. http://dx.doi.org/10.1366/000370210790571954

[5] Scarel, G., Na, J.-S. and Parsons, G.N. (2010) Angular Behavior of the Berreman Effect Investigated in Uniform $\mathrm{Al}_{2} \mathrm{O}_{3}$ Layers Formed by Atomic Layer Deposition. Journal of Physics: Condensed Matter, 22, Article ID: 155401. http://dx.doi.org/10.1088/0953-8984/22/15/155401

[6] Załuzny, M. and Zietkowski, W. (2013) Semiclassical Study of Intersubband Cavity Polaritons: Role of Plasmonic and Radiative Coupling Effects. Physical Review, B88, Article ID: 195408. http://dx.doi.org/10.1103/PhysRevB.88.195408

[7] Fancoeur, M., Mengü M.P. and Vaillon, R. (2010) Local Density of Electromagnetic States within a Nanometric Gap Formed between Two Thin Films Supporting Surface Phonon Polaritons. Journal of Applied Physics, 107, Article ID: 034313. http://dx.doi.org/10.1063/1.3294606

[8] Neubrech, F. and Pucci, A. (2012) Nanoantenna: Plasmon Enhanced Spectroscopies for Biotechnological Applications. Pan Stanford Publishing Pte. Ltd., Singapore City, 297-312.

[9] Vassant, S., Hugonin, J.-P., Marquier, F. and Greffet, J.-J. (2012) Berreman Mode and Epsilon Near Zero Mode. Optics Express, 20, 23971-23977. http://dx.doi.org/10.1364/OE.20.023971

[10] Le Gall, J., Olivier, M. and Greffet, J.-J. (1997) Experimental and Theoretical Study of Reflection and Coherent Thermal Emission by a SiC Grating Supporting a Surface-Phonon Polariton. Physical Review B, 55, Article ID: 10105. http://dx.doi.org/10.1103/PhysRevB.55.10105

[11] Chalopin, Y., Hayoun, M., Volz, S. and Dammak, H. (2014) Surface Enhanced Infrared Absorption in Dielectric Thin Films with Ultra-Strong Confinement Effects. Applied Physics Letters, 104, Article ID: 011905. http://dx.doi.org/10.1063/1.4860989

[12] Kliewer, K.L. and Fuchs, R. (1966) Optical Modes of Vibration in an Ionic Crystal Slab including Retardation. I. Nonradiative Region. Physical Review, 144, 495-503. http://dx.doi.org/10.1103/PhysRev.144.495

[13] Vincent-Johnson, A.J., Schwab, Y., Mann, H.S., Francoeur, M., Hammonds, J.S. and Scarel, G. (2013) Origin of the Low Frequency Radiation Emitted by Radiative Polaritons Excited by Infrared Radiation in Planar $\mathrm{LA}_{2} \mathrm{O}_{3}$ Films. Journal of Physics: Condensed Matter, 25, Article ID: 035901. http://dx.doi.org/10.1088/0953-8984/25/3/035901

[14] Scarel, G., Aita, C.R. and Sklyarov, A.V. (2003) Effect of Substrate Conductivity on Infrared Reflection Spectra of Thin $\mathrm{TiO}_{2}$ Films. Journal of Non-Crystalline Solids, 318, 168-174. http://dx.doi.org/10.1016/S0022-3093(02)01875-6

[15] Scarel, G., Debernardi, A., Tsoutsou, D., Spiga, S., Capelli, S.C., Lamagna, L., Volkos, S.N., Alia, M. and Fanciulli, M. (2007) Vibrational and Electrical Properties of Hexagonal $\mathrm{La}_{2} \mathrm{O}_{3}$ Films. Applied Physics Letters, 91, Article ID: 102901. http://dx.doi.org/10.1063/1.2779108

[16] Vincent-Johnson, A.J., Vasquez, K.A., Bridstrup, J.E., Masters, A.E., Hu, X. and Scarel, G. (2011) Heat Recovery Mechanism in the Excitation of Radiative Polaritons by Broadband Infrared Radiation in Thin Oxide Films. Applied Physics Letters, 99, 131901. http://dx.doi.org/10.1063/1.3643464

[17] Bonera, E., Scarel, G., Fanciulli, M., Delugas, P. and Fiorentini, V. (2005) Dielectric Properties of High- $\kappa$ Oxides: Theory and Experiment for $\mathrm{Lu}_{2} \mathrm{O}_{3}$. Physical Review Letters, 94, Article ID: 027602. http://dx.doi.org/10.1103/PhysRevLett.94.027602

[18] Zhang, X.J., Liu, B.Q., Xu, X.J., Wu, X. and Yuan, R.M. (2014) A Study of the Enhancement in Near-Field Radiative Heat Transfer by Surface Polaritons. Applied Mechanics and Materials, 448-453, 3211-3216.

[19] Ashcroft, N.W. and Mermin, N.D. (1976) Solid State Physics. Saunders College, Philadelphia, 454. 
[20] Fowles, G.R. (1975) Modern Optics. Dover Publications, Inc., New York, 212-217.

[21] Leonhardt, U. (2013) Cloaking of Heat. Nature, 498, 440-441. http://dx.doi.org/10.1038/498440a

[22] Rindler, W. (2006) Relativity. Oxford University Press Inc., New York, 279-281.

[23] Hawking, S.W. (1992) Evaporation of Two-Dimensional Black Holes. Physical Review Letters, 69, 406-409. http://dx.doi.org/10.1103/PhysRevLett.69.406

[24] Braunstein, S.L. and Patra, M.K. (2011) Black Hole Evaporation Rates without Spacetime. Physical Review Letters, 107, Article ID: 071302. http://dx.doi.org/10.1103/PhysRevLett.107.071302

[25] Łach, G., DeKieviet, M. and Jentschura, U.D. (2012) Enhancement of Blackbody Friction Due to the Finite Lifetime of Atomic Levels. Physical Review Letters, 108, Article ID: 043005. http://dx.doi.org/10.1103/PhysRevLett.108.043005

[26] Unruh, W.G. (1981) Experimental Black-Hole Evaporation? Physical Review Letters, 46, 1351-1353. http://dx.doi.org/10.1103/PhysRevLett.46.1351

[27] Dimitrov, L.F. (2011) Biological Membranes Are Nanostructures That Require Internal Heat and Imaginary Temperature as New, Unique Physiological Parameters Related to Biological Catalysis. Cell Biochemistry and Biophysics, 59, 133-146. http://dx.doi.org/10.1007/s12013-010-9134-8 